\title{
A prospective cohort study of latent tuberculosis in adult close contacts of active pulmonary tuberculosis patients in Korea
}

\author{
Sun Hyo Park ${ }^{1}$, Seung Jun Lee ${ }^{2}$, Yu Ji Cho ${ }^{2,3}$, Yi Yeong Jeong ${ }^{2,3}$, Ho Cheol Kim²,3 Jong Deog Lee ${ }^{2,3}$, \\ Hee Jin $\mathrm{Kim}^{4}$, and Dick Menzies ${ }^{5}$
}

\begin{abstract}
${ }^{1}$ Department of Internal Medicine, Dongnam Institute of Radiological \& Medical Sciences Cancer Center, Busan; ${ }^{2}$ Department of Internal Medicine, Gyeongsang National University School of Medicine, Jinju; ${ }^{3}$ Gyeongsang Institute of Health Sciences, Gyeongsang National University, Jinju; ${ }^{4}$ Korean Institute of Tuberculosis, Seoul, Korea; ${ }^{5}$ Montreal Chest Institute, McGill University, Montreal, QC, Canada
\end{abstract}

Received: April 9, 2015

Revised : May 28, 2015

Accepted: June 15, 2015

\section{Correspondence to}

Ho Cheol Kim, M.D.

Department of Internal Medicine, Gyeongsang National University School of Medicine, 15 Jinju-daero 816beon-gil, Jinju 52727, Korea

Tel: $+82-55-750-8684$

Fax: $+82-55-750-8618$

E-mail: hockkim@gnu.ac.kr
Background/Aims: The objective of this prospective study was to evaluate the diagnosis and treatment of latent tuberculosis infection (LTBI) in adult close contacts of active pulmonary tuberculosis (TB) patients in Korea.

Methods: Adult close contacts of active pulmonary TB patients were recruited at a regional tertiary hospital in Korea. The participants were tested for LTBI using the tuberculin skin test (TST) and/or QuantiFERON-TB Gold (QFT-G) test. LTBI patients, who consented to treatment, were randomly assigned to receive isoniazid for 9 months (9INH) or rifampin for 4 months (4RIF).

Results: We examined 189 adult close contacts ( $>18$ years) of 107 active pulmonary TB patients. The TST and QFT-G were positive ( $\geq 10 \mathrm{~mm})$ in $75 / 183(39.7 \%)$ and $45 / 118$ (38.1\%) tested participants, respectively. Among 88 TST or QFT-G positive LTBI participants, 45 participants were randomly assigned to receive 4 RIF ( $\mathrm{n}$ $=21)$ or $9 \mathrm{INH}(\mathrm{n}=24)$, respectively. The average treatment duration for the $4 \mathrm{RIF}$ and $9 \mathrm{INH}$ groups was $3.3 \pm 1.3$ and $6.1 \pm 2.7$ months, respectively. Treatment was completed in 25 participants ( $4 \mathrm{RIF}, \mathrm{n}=16$; $9 \mathrm{INH}, \mathrm{n}=9$ ). LTBI participants who accepted treatment were more likely to be women and have more cavitary lesions on the chest radiographs of index cases and positive TST and QFT-G results compared to those who refused treatment.

Conclusions: About $40 \%$ of adult close contacts of active pulmonary TB patients had LTBI; about $50 \%$ of these LTBI participants agreed to treatment.

Keywords: Latent tuberculosis; Tuberculin test; Tuberculosis, pulmonary; Isoniazid; Rifampin

\section{INTRODUCTION}

Korea has an intermediate tuberculosis (TB) burden, and the annual incidence of new active TB cases in this country is estimated to be 71.3 in 100,000 people $[1,2]$. Recently, there have been reports regarding the latent tuberculosis infection (LTBI) rate among Korean healthcare workers and individuals who have been in close contact with active pulmonary TB patients [3-6].

People exposed to TB are at risk of acquiring either LTBI or active TB. Although those with LTBI do not show signs, symptoms, or radiographic evidence of TB disease [7], they have a 10\% lifetime risk of developing active TB. Half of all active TB cases occur within the first 2 years following T'B exposure [8]. Therefore, early diagnosis and treatment of LTBI could be a useful strat- 
egy for reducing the long-term burden of TB, especially among people who have been in close contact with active TB patients.

According to the Korean guidelines for TB reported in 2014, the LTBI test is recommended for close contacts of active pulmonary TB patients and treatment should be considered for those $<35$ years of age [9]. The diagnosis and treatment of LTBI in Korean adult close contacts of active pulmonary TB patients have not yet been reported. Here, we have reported our experience regarding the diagnosis and treatment of adults who contracted LTBI from close contact with active TB patients.

\section{METHODS}

\section{Subjects and study design}

The data were collected during an international multicenter trial of LTBI entitled, "A randomized clinical trial comparing 4 RIF vs. 9INH for treatment of latent tuberculosis infection: effectiveness" (registration number; NCToo931736). The study protocol and inclusion and exclusion criteria of participants are described in the website (https://clinicaltrials.gov/ct2/show/study/NCToo931736). According to study protocol, the inclusion criterion for treatment was a tuberculin skin test (TST) reaction of $\geq$ $5 \mathrm{~mm}$ for close contacts of active pulmonary TB patients.

This current study was conducted at a single regional tertiary hospital in Korea, which was part of the international multicenter trial. This study was approved by the Institutional Review Board of Gyeongsang National University Hospital, Korea (IRB No. GNUHIRB-2009-042). Informed consent was obtained from the enrolled participants.

From October 2009 to August 2013, we recruited individuals (aged $>18$ years), who were considered to be close contacts of active pulmonary TB patients. These individuals were asked to be volunteers for the LTBI test at the Gyeongsang National University Hospital, Korea. A "close contact" was defined as a person who had contact, for at least 4 hours/week, with a patient(s) with active pulmonary TB during an infectious period, and was confirmed by an acid-fast bacilli (AFB) smear and/or AFB culture test. We excluded health care workers, those diagnosed with active TB after chest radiography, and those who were in contact with patients with drug-re- sistant TB as indicated by the drug susceptibility profile. Participants underwent a TST and/or QuantiFERON-TB Gold (QFT-G, Cellestis Ltd., Carnegie, Australia) test after signing informed consent. If both tests were conducted during the same day, the TST was performed first. We assessed risk factors for contracting LTBI, including age, sex, history of bacilli-Calmette-Guerin (BCG) vaccination, history of pulmonary TB, cohabiting with a TB patient in the same house or room, presence of cough, AFB smear status of the index case, and cavitary lesions on the chest radiographs of the index case.

\section{TST and QFT-G}

The TST was performed according to the Mantoux method (Statens Serum Institute, Copenhagen, Denmark). The participants were injected intradermally with $0.1 \mathrm{~mL}$ of tuberculin purified protein derivative RT 23 SSI (2TU) by a trained research nurse, and the transverse diameter $(\mathrm{mm})$ of the induration at the injection site (inner forearm) was measured 48 to 72 hours after injection [10,11]. The QFT-G tests were performed by a specialized laboratory company (Samkwang Medical Laboratories, Seoul, Korea) according to the manufacturer's instructions. We incubated whole blood at $37^{\circ} \mathrm{C}$ for 16 to 24 hours with TB-specific antigens containing an early-secreted antigenic target 6 protein and culture filtrate protein 10, and then measured the concentration of the released interferon gamma in the supernatant solution using an enzyme-linked-immunosorbent serologic assay [12,13].

\section{Treatment of LTBI}

In this study, LTBI was defined as a positive result for either TST ( $\geq 10 \mathrm{~mm}$ ) or QFT-G, and participants who tested positive for LTBI were randomly allocated to receive isoniazid for 9 months ( $9 \mathrm{INH}$; 9 months of $5 \mathrm{mg}$ / $\mathrm{kg}$ isoniazid up to $300 \mathrm{mg} /$ day) or rifampin for 4 months (4RIF; 4 months of $10 \mathrm{mg} / \mathrm{kg}$ rifampin up to $600 \mathrm{mg} /$ day). Participants who showed resistance to isoniazid or rifampin during the drug sensitivity test were excluded from the study. Participants were allowed to withdraw from treatment if they choose to do so. We also compared LTBI patients who accepted and refused treatment. 
Table 1. Clinical characteristics of participants who underwent the tuberculin skin test and/or QuantiFERON-TB Gold test

\begin{tabular}{|c|c|c|c|c|}
\hline Characteristic & All & TST & QFT-G & TST and QFT-G \\
\hline Number & 189 & 183 & 118 & 112 \\
\hline Age, yr & $48.7 \pm 15.8(18-68)$ & $48.5 \pm 15.6$ & $50.4 \pm 14.6$ & $50.1 \pm 14.4$ \\
\hline Sex, male:female & $59: 130$ & $57: 126$ & $39: 79$ & $37: 75$ \\
\hline BCG vaccination scar & $170(89.9)$ & $166(90.7)$ & $104(88.1)$ & $100(89.2)$ \\
\hline History of pulmonary TB & $15(7 \cdot 9)$ & $15(8.2)$ & $9(7.6)$ & $9(8)$ \\
\hline Existence of comorbid illness & $55(29.1)$ & $53(28.9)$ & $28(23.7)$ & $26(23.2)$ \\
\hline \multicolumn{5}{|l|}{ Smoking status } \\
\hline Current & $18(9.5)$ & $17(9 \cdot 3)$ & $14(11.9)$ & $13(9.8)$ \\
\hline Never smoker & $58(30.7)$ & $57(31.1)$ & $44(37 \cdot 3)$ & $43(38.3)$ \\
\hline Household contact & $140(74.1)$ & $135(73.8)$ & $86(72.9)$ & $81(72.3)$ \\
\hline Stayed at the same room & $79(41.8)$ & $76(41.5)$ & $51(43.2)$ & $48(42.8)$ \\
\hline $\begin{array}{l}\text { Sputum or bronchial washing fluid } \\
\text { AFB smear positive in index cases }\end{array}$ & $151(79.9)$ & $146(79.8)$ & $92(77 \cdot 9)$ & $87(77.6)$ \\
\hline Presence of cough in index cases & $122(64.6)$ & $118(64 \cdot 5)$ & $80(67.8)$ & $76(67.8)$ \\
\hline $\begin{array}{l}\text { Cavitary lesion on the chest radiograph in } \\
\text { index cases }\end{array}$ & $81(42.9)$ & $79(43.2)$ & $49(41.5)$ & $47(41.9)$ \\
\hline
\end{tabular}

Values are presented as mean $\pm \mathrm{SD}$ or number (\%).

TB, tuberculosis; TST, tuberculin skin test; QFT-G, QuantiFERON-TB Gold; BCG, Bacille-Calmette-Guerin; AFB, acid-fast bacilli.

\section{Statistical analysis}

Descriptive data were presented as numbers and percentages; continuous data were indicated by mean \pm standard deviation or median (range). Categorical variables were compared using the chi-square test or Fisher exact test, as appropriate. Continuous variables were compared using the parametric $t$ test or the Mann-Whitney $U$ test. Differences were considered statistically significant if $p<0.05$. Statistical analyses were performed using SPSS version 18.0 (SPSS Inc., Chicago, IL, USA).

\section{RESULTS}

\section{Characteristics of study participants}

We identified 348 adults who had close contact with 128 active pulmonary TB patients who were willing to test for LTBI with the TST and/or QFT-G. We excluded health care workers $(n=61)$ and those aged $<18$ years $(n=$ 7). Individuals were also excluded if the TB patient with whom they had close contact with had the following: (1) no Mycobacterium tuberculosis (MTB) growth $(\mathrm{n}=31$ ) on their sputum or bronchial washing fluid samples; (2) positive test for mono- or multi-drug resistance $(n=21)$; and (3) no documented results of a drug sensitivity test for MTB ( $n=40)$ (Fig. 1). After these exclusions, we enrolled 189 participants who were close contacts of 107 active TB patients. The mean number of tested participants per active TB patient was $1.8 \pm 1.2$ (range, 1 to 7 ). The number of participants who underwent the TST, QFT-G, and both TST and QFT-G tests were 183, 118, and 112 participants, respectively. Seventy-six participants were tested with TST and QFT-G on the same day, and the mean time interval for undergoing both tests was $3.1 \pm 2.2$ days (range, 1 to 14 ). Two participants were tested with the QFT-G 7 and 14 days after the TST.

The mean age of participants was $48.7 \pm 15.7$ years; 130 (68.8\%) were women and 140 were household contacts (74.1\%). Most of the participants $(\mathrm{n}=170,89.9 \%)$ had a scar from BCG vaccination (90.7\% and $88.1 \%$ in the TST and QFT-G groups, respectively). A history of pulmonary TB was noted in $8.2 \%$ and $7.6 \%$ of the participants in the TST and QFT-G groups, respectively (Table 1). Comorbid diseases, including hypertension, diabetes, cardiovascular, chronic liver, and thyroid disease, were reported in $28.9 \%$ and $23.7 \%$ of participants in the TST 


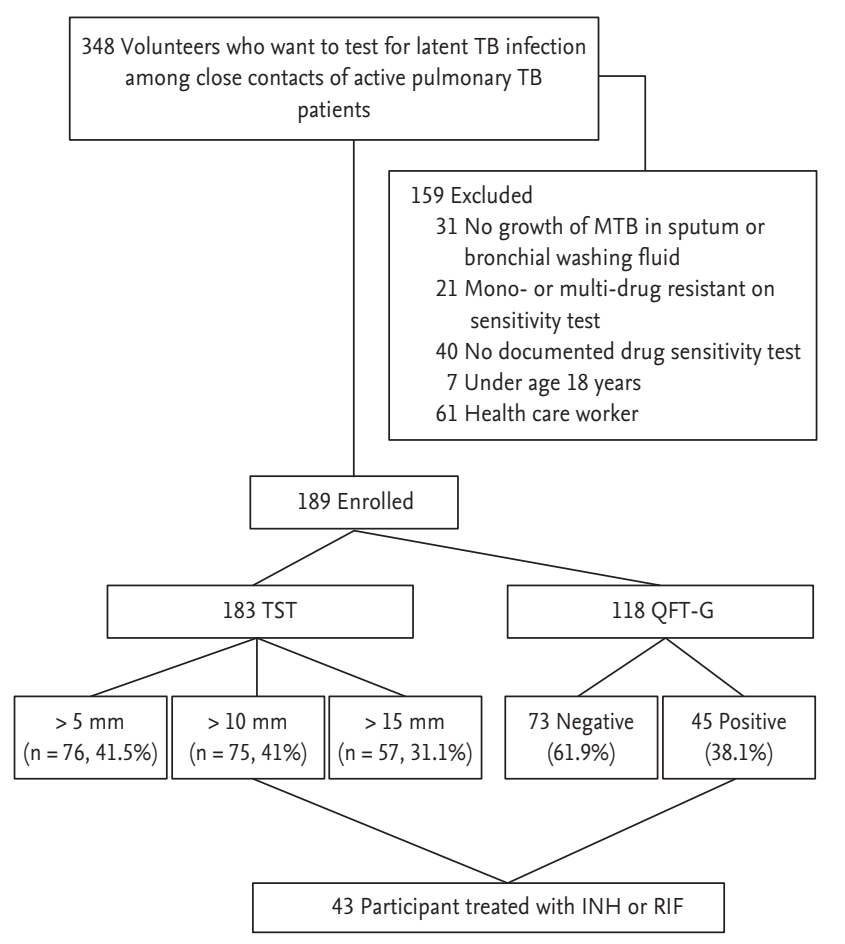

Figure 1. Flow chart of the study. Diagnosis of latent tuberculosis (TB) assessed by the tuberculin skin test (TST) and/ or QuantiFERON-TB Gold (QFT-G) test. MTB, Mycobacterium tuberculosis; INH, isoniazid; RIF, rifampin.

and QFT-G groups, respectively. More than half of the participants in both the TST and QFT-G groups were household close contacts $(73.8 \%$ and $72.9 \%$ in the TST and QFT-G groups, respectively) and resided in the same room (41.5\% and $43.2 \%$ in the TST and QFT-G groups, respectively). In index cases, the rate of positive AFB smears obtained from the sputum or bronchial washing fluid in active pulmonary TB patients was 79.8\% and $77.9 \%$ in the TST and QFT-G groups, respectively. Coughing as a respiratory symptom was observed in about $60 \%$ of active pulmonary TB patients. About $40 \%$ of active pulmonary TB patients were observed to have cavitary lesions in their chest radiographs.

\section{TST and QFT-G}

Seventy-five of the 183 participants who underwent TST ( $\geq 10 \mathrm{~mm}, 40.9 \%$ ) and 45 of the 118 participants who underwent QFT-G testing (41.0\%) had positive results. There were no other significant differences in the clinical characteristics of TST-positive and TST-negative participants, including the mean age, proportion of participants with visible BCG vaccination scars, histo-

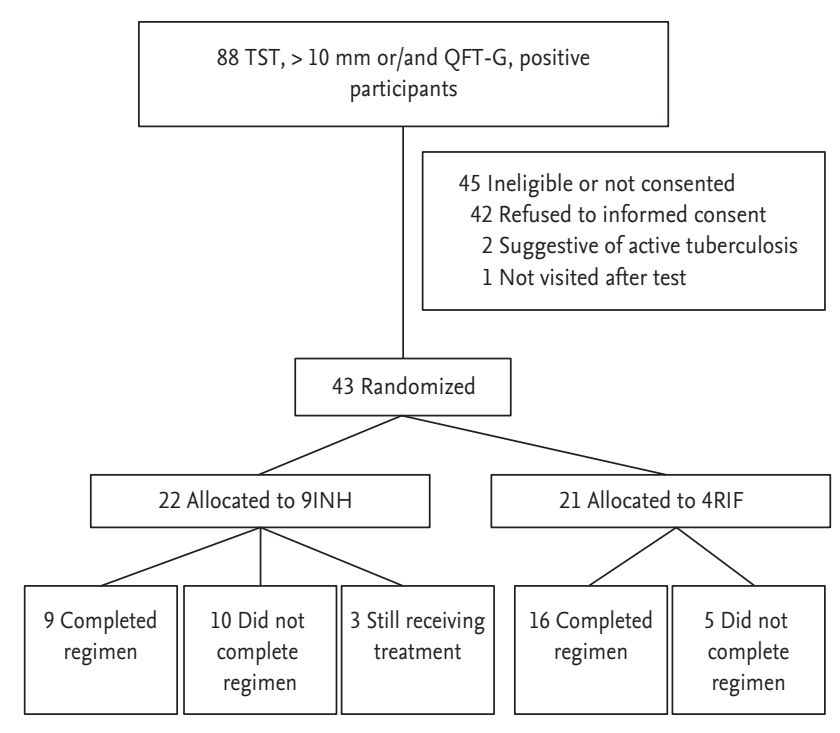

Figure 2. Flow chart of the study. Treatment of latent tuberculosis patients assessed by the tuberculin skin test (TST) and/or QuantiFERON-TB Gold (QFT-G) test using isoniazid for 9 months $(9 \mathrm{INH})$ or rifampin for 4 months (4RIF).

ry of TB, existence of comorbid illnesses, proportion of participants who had never smoked, household contacts (or residing in the same room), AFB smear status, presence of cough, or chest radiograph-confirmed cavitary lesion(s) in the index cases with LTBI. Similar findings were also observed in QFT-G-positive and QFT-G-negative participants (Table 2).

\section{Treatment of LTBI}

Among the 88 participants with TST or QFT-G positive results, 43 agreed to treatment. Forty-two of the 45 participants did not consent to treatment, two had signs of active TB on their chest radiograph, and one did not visit the hospital after TST/QFT-G testing. There were no differences in age, smoking status, history of TB, presence of cough in index cases, and household contacts (or residing in the same room) between those who agreed to and refused treatment. However, a higher percentage of women ( $76.7 \%$ vs. $60.0 \%$, respectively) and those with cavitary lesions on chest radiograph in index cases ( $55.8 \%$ vs. $24.4 \%$, respectively) accepted rather than refused treatment. Among the 63 participants who underwent testing for both TST and QFT-G, a positive result for both tests was more common in those who accepted treatment compared to those who refused treatment (62.5\% vs. $36.4 \%$, respectively) (Table 3). Twenty of 
Table 2. Comparison of clinical characteristics in participants with positive and negative tuberculin skin test or QuantiFERON-TB Gold test results

\begin{tabular}{|c|c|c|c|c|c|c|}
\hline \multirow[b]{2}{*}{ Characteristic } & \multicolumn{3}{|c|}{ TST } & \multicolumn{3}{|c|}{ QFT-G } \\
\hline & $\begin{array}{l}\text { Positive }(+) \\
\geq 10 \mathrm{~mm}\end{array}$ & $\begin{array}{l}\text { Negative }(-) \\
\quad<10 \mathrm{~mm}\end{array}$ & $p$ value & Positive (+) & Negative (-) & $p$ value \\
\hline Number & 75 & 108 & & 45 & 73 & \\
\hline Age, yr & $48.9 \pm 13.8$ & $48.1 \pm 16.8$ & 0.725 & $51.7 \pm 15.5$ & $49 \cdot 5 \pm 14 \cdot 1$ & 0.431 \\
\hline Sex, male:female & $28: 47$ & 29:79 & 0.132 & $15: 30$ & $24: 49$ & 1.000 \\
\hline QFT-G (+) & 32 & 9 & 0.000 & & & \\
\hline $\mathrm{TST} \geq 10 \mathrm{~mm}$ & & & & 32 & 24 & 0.000 \\
\hline BCG vaccination scar & $68(90.7)$ & $98(90.7)$ & 0.986 & $38(84.4)$ & $66(90.4)$ & 0.385 \\
\hline History of pulmonary TB & $9(12.0)$ & $6(5.6)$ & 0.170 & $5(11.1)$ & $4(5 \cdot 5)$ & 0.299 \\
\hline Existence of comorbid illness & $20(26.7)$ & $23(21.3)$ & 0.399 & $15(33 \cdot 3)$ & $13(17.8)$ & 0.074 \\
\hline \multicolumn{7}{|l|}{ Smoking status } \\
\hline Current & $8(10.6)$ & $9(8.3)$ & 0.233 & $5(11.1)$ & $9(12.3)$ & 0.153 \\
\hline Never smoker & $17(22.7)$ & $40(37)$ & 0.115 & $13(28.9)$ & $31(42.5)$ & 0.159 \\
\hline Household contact & $58(77 \cdot 3)$ & $77(71.3)$ & 0.397 & $35(77 \cdot 7)$ & $51(69.8)$ & 0.399 \\
\hline Stayed at the same room & $33(44)$ & $43(39.8)$ & 0.648 & $19(44.2)$ & $32(43.8)$ & 0.864 \\
\hline $\begin{array}{l}\text { Sputum or bronchial washing fluid } \\
\text { AFB smear positive in index cases }\end{array}$ & $63(84)$ & $83(76.8)$ & 0.266 & $39(86.6)$ & $53(72.6)$ & 0.109 \\
\hline Presence of cough in index cases & $51(68)$ & $68(62.9)$ & 0.436 & $29(64.4)$ & $51(69.8)$ & 0.550 \\
\hline $\begin{array}{l}\text { Cavitary lesion on the chest } \\
\text { radiograph in index cases }\end{array}$ & $29(38.7)$ & $50(46.2)$ & 0.363 & $15(33.3)$ & $34(46.6)$ & 0.181 \\
\hline
\end{tabular}

Values are presented as mean \pm SD or number (\%).

TB, tuberculosis; TST, tuberculin skin test; QFT-G, QuantiFERON-TB Gold; BCG, Bacille-Calmette-Guerin; AFB, acid-fast bacilli.

the 43 participants who agreed to treatment had positive results for both TST and QFT-G, while eight and six participants tested positive for only TST and QFT-G, respectively. Those who consented to treatment were randomly assigned to receive $9 \mathrm{INH}(\mathrm{n}=22)$ or $4 \mathrm{RIF}(\mathrm{n}=21)$. The median duration of treatment for participants who underwent $9 \mathrm{INH}$ and ${ }_{4} \mathrm{RIF}$ was 6 months (range, 1 to 9) and 4 months (range, 1 to 4 ), respectively. Nine of the 22 patients in the $9 \mathrm{INH}$ group and 16 of the 21 participants in the 4 RIF group completed treatment (Fig. 2).

\section{DISCUSSION}

To the best of our knowledge, this was the first study in which the diagnoses and treatment experiences have been reported for adults who contracted LTBI after having close contact with active pulmonary TB patients in
Korea. About $40 \%$ of adult close contacts of active pulmonary TB patients were diagnosed as having LTBI, and about $50 \%$ of these LTBI participants agreed to treatment. Based on the Korean guideline of $\geq 10 \mathrm{~mm}$ for TST positivity, $38.4 \%$ of our participants tested positive for LTBI. The incidence of a positive TST result for close contacts of active TB patients in Korea is variable. Previously reported incidence rates for a positive TST result include $47.1 \%(n=90 / 206)$ by Park [6], 71\% by Kang et al. [14], and $14.7 \%(n=270 / 1826)$ during a school TB outbreak by Song et al. [5] In other countries, the incidence rate of LTBI among close contacts of active TB patients, as assessed by TST, also varied considerably from $27.9 \%$ to $93.0 \%$ [15].

The interferon gamma release assay (IGRA) has been known as an accepted specific test to detect LTBI, and therefore, it is widely used in Korea. In previous studies that involved the assessment of the incidence of LTBI in 
Table 3. Comparison of treatment acceptance and refusal in latent tuberculosis participants $(n=88)$

\begin{tabular}{|c|c|c|c|}
\hline Characteristic & Accepted & Refused & $p$ value \\
\hline Number & 43 & 45 & \\
\hline Age, yr & $50.9 \pm 12.9$ & $47 \cdot 4 \pm 16.1$ & 0.265 \\
\hline Sex, male:female & $10: 33$ & $22: 33$ & 0.007 \\
\hline QFT-G (+) & $26(76.5)$ & $19(54 \cdot 3)$ & 0.053 \\
\hline $\mathrm{TST} \geq 10 \mathrm{~mm}$ & $37(90.2)$ & $38(88.4)$ & 0.782 \\
\hline QFT-G $(+)$ and TST $\geq 10 \mathrm{~mm}^{\mathrm{a}}$ & $20(62.5)$ & $12(36.4)$ & 0.035 \\
\hline BCG vaccination scar & $37(86)$ & $42(93 \cdot 3)$ & 0.259 \\
\hline History of pulmonary TB & $6(14.0)$ & $3(6.7)$ & 0.259 \\
\hline Existence of comorbid illness & $13(30.2)$ & $12(26.7)$ & 0.711 \\
\hline \multicolumn{4}{|l|}{ Smoking status } \\
\hline Current & $5(11.6)$ & $5(11.1)$ & 0.340 \\
\hline Household contact & $10(23.3)$ & $10(22.2)$ & \\
\hline Stayed at the same room & $20(46.5)$ & $18(40.0)$ & 0.538 \\
\hline Hospital admission of index cases & $31(72.1)$ & $33(73 \cdot 3)$ & 1.000 \\
\hline Sputum or bronchial washing fluid AFB smear positive in index cases & $36(83.7)$ & $39(86.7)$ & 0.697 \\
\hline Presence of cough in index cases & $27(62.8)$ & $32(71.1)$ & 0.407 \\
\hline Cavitary lesion on the chest radiograph in index cases & $24(55.8)$ & $11(24 \cdot 4)$ & 0.003 \\
\hline
\end{tabular}

Values are presented as mean $\pm \mathrm{SD}$ or number (\%).

QFT-G, QuantiFERON-TB Gold; TST, tuberculin skin test; BCG, Bacille-Calmette-Guerin; TB, tuberculosis; AFB, acid-fast bacilli.

${ }^{\mathrm{a} B o t h}$ TST and QFT were performed in 112 volunteers.

Korea using IGRA, a positive QFT-G result was observed in $11.1 \%(n=203 / 1826)$ of participants who had close contact with active TB patients [5]. The percentages of positive IGRA results observed in previous studies ( $44 \%$ and $53.5 \%$ of participants in the studies by Kang et al. [14] and Jo et al. [16], respectively) were slightly higher compared to our study findings. However, the incidence of LTBI, based on positive IGRA results in our study, was considerably higher compared to a previous study conducted among casual contacts in Korea with no identifiable risks of infection (IGRA positivity of < $5 \%$ to $10 \%$ ) [14]. These findings indicate higher risks of LTBI in close contacts of active pulmonary T'B patients.

A positive QFT was associated with a positive smear and cough index of the TB source case and a prolonged contact time in studies that involved the evaluation of factors associated with acquiring LTBI in close contacts of active TB patients [17-19]. In addition, residing in the same room as the index case was associated with a positive QFT [20]. However, a positive TST result in close contacts of active TB patients was not associated with a positive smear during the presence of cough and delayed diagnosis [21]. In our previous reports, we showed that there were no significant factors associated with a positive QFT and previous history of TB, and household contact was associated with a positive TST result in close contacts of active TB patients [22].

Approximately $60 \%$ of our participants who tested positive for LTBI refused treatment. We believe that the relatively high treatment refusal rate was due to the following factors: (1) participants were reluctant to receive treatment for an infection if they did not experience any symptoms; (2) participants were also concerned about the adverse effects of anti-TB medication; (3) there was no evidence to prove that the infection improved or that the participant was cured; and (4) under the Korean guidelines, treatment was not recommended in participants who were aged $>35$ years and had close contact with active TB patients. Interestingly, among the LTBI participants, a higher percentage of women, those with cavitary lesions on the chest radiographs of index cases, and those with positive results for both TST and QFT-G 
accepted treatment compared to those who refused treatment. Women and those who had cavitary lesions on their chest radiographs were more concerned or anxious about having an infection compared to other participants. Physicians were more prone to diagnose LT'BI in participants who had close contact with active TB patients and a positive result for both TST and QFT-G compared to those who had a positive result for only TST or QFT-G. These explanations may lead to a higher probability of accepting LTBI treatment.

Bacilli seem to have low metabolic activity in LTBI and consequently, LTBI should be treated for extended periods in order to be effective. A lengthy treatment regimen along with the asymptomatic nature of LTBI can negatively affect treatment completion [23]. In this study, about half the participants completed their treatment regimen. The acceptance and discontinuation rates of LTBI treatment vary. Only $26 \%$ of people identified with LTBI initiated their treatments in a study conducted at public health clinics in the United States; however, only $53 \%$ of them completed the treatment course [24]. Horsburgh [8] observed completion rates of $69 \%$ and $45 \%$ for 4 RIF and $9 \mathrm{INH}$ among high-risk populations at public clinics. The completion rate was higher for 4 RIF compared to $9 \mathrm{INH}$ in this study. However, a longer follow-up duration is needed to determine the differences in completion rates because different treatment durations can affect the completion rate of each regimen.

In conclusion, this was the first study regarding the diagnoses and treatments of LTBI in adult close contacts of patients with active pulmonary TB in Korea. The Korean guidelines regarding LTBI treatment should be considered to reduce the incidence of TB among these individuals. Furthermore, studies should also be conducted to verify the efficacy of LTBI treatment and its adverse effects in close contacts of active T'B patients.

\section{KEY MESSAGE}

1. Among 189 adult close contacts with active pulmonary tuberculosis patients, the positive rate for tuberculin skin test (TST) and QuantiFERON-TB Gold (QFT-G) was approximately 40\%.

2. Among 88 participants with latent tuberculosis infection, a high percentage of women, those with cavitary lesions on the chest radiographs of index cases, and those with positive TST and QFT-G accepted treatment.

\section{Conflict of interest}

No potential conflict of interest relevant to this article was reported.

\section{Acknowledgments}

The research was funded by the Canadian Institutes of Health Research (NCToo931736).

\section{REFERENCES}

1. Raviglione MC, O'Brien RJ. Tuberculosis. In: Longo DL, Fauci AS, Kasper DL, Hauser SL, Jameson JL, Loscalzo J, eds. Harrison's Principles of Internal Medicine. 18th ed. New York: McGraw-Hill, 2012:1340-1359.

2. World Health Organization. Global Tuberculosis Report 2013. Geneva: World Health Organization, 2013.

3. Lee SJ, Kim HS, Ma JE, et al. Tuberculin skin test and QuantiFERON-TB Gold assay before and after treatment for latent tuberculosis infection among health care workers in local tertiary hospital. Tuberc Respir Dis 2007;62:270-275.

4. Lee K, Han MK, Choi HR, et al. Annual incidence of latent tuberculosis infection among newly employed nurses at a tertiary care university hospital. Infect Control Hosp Epidemiol 2009;30:1218-1222.

5. Song S, Jeon D, Won Kim J, et al. Performance of confirmatory interferon-gamma release assays in school TB outbreaks. Chest 2012;141:983-988.

6. Park JS. Prevalence and risk factors for mycobacterium tuberculosis infection among contacts of pulmonary tuberculosis patients. Tuberc Respir Dis 2012;72:140-148.

7. National Center for HIV/AIDS, Viral Hepatitis, STD, and TB Prevention (U.S.). Division of Tuberculosis Elimination; New Jersey Medical School Global Tuberculosis Institute. Latent Tuberculosis Infection: A Guide for Primary Health Care Providers. Atlanta: Centers for Disease Control and Prevention, 2010.

8. Horsburgh CR Jr. Priorities for the treatment of latent tuberculosis infection in the United States. N Engl J Med 2004;350:2060-2067.

9. Lee SH. Diagnosis and treatment of latent tuberculosis 
infection. Tuberc Respir Dis (Seoul) 2015;78:56-63.

10. Sokal JE. Editorial: measurement of delayed skin-test responses. N Engl J Med 1975;293:501-502.

11. Choi JC, Shin JW, Kim JY, Park IW, Choi BW, Lee MK. The effect of previous tuberculin skin test on the follow-up examination of whole-blood interferon-gamma assay in the screening for latent tuberculosis infection. Chest 2008;133:1415-1420.

12. Brock I, Weldingh K, Lillebaek T, Follmann F, Andersen P. Comparison of tuberculin skin test and new specific blood test in tuberculosis contacts. Am J Respir Crit Care Med 2004;170:65-69.

13. Mori T, Sakatani M, Yamagishi F, et al. Specific detection of tuberculosis infection: an interferon-gamma-based assay using new antigens. Am J Respir Crit Care Med 2004;170:59-64.

14. Kang YA, Lee HW, Yoon HI, et al. Discrepancy between the tuberculin skin test and the whole-blood interferon gamma assay for the diagnosis of latent tuberculosis infection in an intermediate tuberculosis-burden country. JAMA 2005;293:2756-2761.

15. Morrison J, Pai M, Hopewell PC. Tuberculosis and latent tuberculosis infection in close contacts of people with pulmonary tuberculosis in low-income and middle-income countries: a systematic review and meta-analysis. Lancet Infect Dis 2008;8:359-368.

16. Jo KW, Jeon K, Kang YA, et al. Poor correlation between tuberculin skin tests and interferon-gamma assays in close contacts of patients with multidrug-resistant tuberculosis. Respirology 2012;17:1125-1130.

17. Diel R, Loddenkemper R, Meywald-Walter K, Gottschalk
R, Nienhaus A. Comparative performance of tuberculin skin test, QuantiFERON-TB-Gold In Tube assay, and T-Spot.TB test in contact investigations for tuberculosis. Chest 2009;135:1010-1018.

18. Shanaube K, Hargreaves J, Fielding K, et al. Risk factors associated with positive QuantiFERON-TB Gold In-Tube and tuberculin skin tests results in Zambia and South Africa. PLoS One 2011;6:e18206.

19. Arend SM, Thijsen SF, Leyten EM, et al. Comparison of two interferon-gamma assays and tuberculin skin test for tracing tuberculosis contacts. Am J Respir Crit Care Med 2007;175:618-627.

20. Adetifa IM, Lugos MD, Hammond A, et al. Comparison of two interferon gamma release assays in the diagnosis of Mycobacterium tuberculosis infection and disease in The Gambia. BMC Infect Dis 2007;7:122.

21. de Souza-Galvao ML, Latorre I, Altet-Gomez N, et al. Correlation between tuberculin skin test and IGRAs with risk factors for the spread of infection in close contacts with sputum smear positive in pulmonary tuberculosis. BMC Infect Dis 2014;14:258.

22. Lee SJ, Lee SH, Kim YE, et al. Risk factors for latent tuberculosis infection in close contacts of active tuberculosis patients in South Korea: a prospective cohort study. BMC Infect Dis 2014;14:566.

23. Smith BM, Menzies D. Treatment of latent TB: first do no harm. Expert Rev Anti Infect Ther 2011;9:491-493.

24. Goswami ND, Gadkowski LB, Piedrahita C, et al. Predictors of latent tuberculosis treatment initiation and completion at a U.S. public health clinic: a prospective cohort study. BMC Public Health 2012;12:468 